\title{
Nahm's Equations and Hyperkähler Geometry
}

\author{
Andrew S. Dancer \\ Peterhouse, Cambridge, CB2 1RD, United Kingdom and DAMTP, Silver Street, Cambridge, \\ CB3 9EW, United Kingdom \\ Address from 1st October 1993: Max-Planck-Institut für Mathematik, Gottfried-Claren-Strasse 26, \\ D-53225 Bonn, Germany
}

Received: 17 December 1991

\begin{abstract}
The geometry of certain moduli spaces of solutions to Nahm's equations is studied, and a family of gravitational instantons is shown to arise as a deformation of the Atiyah-Hitchin manifold.
\end{abstract}

\section{Introduction}

Considerable effort has been devoted to the study of moduli spaces of solutions to the self-dual Yang-Mills equations and their dimensional reductions. One reason for this is that such moduli spaces can often be naturally endowed with a hyperkähler structure. This consists of a metric and three covariant constant complex structures satisfying the quaternionic multiplication relations. Hyperkähler manifolds are necessarily $4 n$ dimensional, where $n$ is an integer, and their holonomy is contained in $S p(n)$. The possible existence of such manifolds was implicit in Berger's classification of the groups which could arise as holonomy groups of non-symmetric Riemannian manifolds: however nontrivial examples of dimension higher than four were not known until the work of Calabi [C]. Four-dimensional hyperkähler manifolds are, in the terminology of physics, examples of gravitational instantons.

In this paper, we shall introduce a twelve-dimensional moduli space $M^{12}$ of solutions to Nahm's equations, a nonlinear system of ordinary differential equations arising as a reduction of the self-dual Yang-Mills equations. The manifold $M^{12}$ admits a hyperkähler structure, and is acted on isometrically by $U(2)$ and $\operatorname{Spin}(3)$. The $U(2)$ action is triholomorphic (preserves the Kähler structures) while the action of Spin(3) permutes the Kähler structures. The hyperkähler quotient of $M^{12}$ by the centre of $U(2)$ is an eight-dimensional hyperkähler manifold $M^{8}$ with an isometric $S U(2) \times S O(3)$ action. We show that $M^{8}$ is homeomorphic to $\mathbb{R}^{5} \times S U(2) / \mathbf{Z}_{2}$ and calculate the $L^{2}$ metric on $M^{8}$ and on the quotient $N^{5}=M^{8} / S U(2)$. We also study a totally geodesic submanifold $\Sigma$ of $M^{8}$ which represents axisymmetric solutions to the Nahm equations. Finally, we obtain a family of hyperkähler four-manifolds as hyperkähler quotients of $M^{8}$ by a circle subgroup of $S U(2)$. These manifolds, which we believe 
to be new examples of gravitational instantons, arise as deformations of the moduli space of charge two $S U(2)$ monopoles studied in [AH].

It is well known $[\mathrm{H} 1, \mathrm{~N}]$ that Nahm data with appropriate boundary conditions gives rise, via the ADHM-Nahm construction, to monopoles on $\mathbb{R}^{3}$. In another paper [D1] we have shown that the ADHM-Nahm procedure associates to points of our moduli space $S U(3)$ monopoles with symmetry breaking to $U(2)$. Now Manton [M] has observed that geodesic motion on a moduli space of monopoles should, at least at low energies, give a good approximation to monopole dynamics. Assuming that the transform from Nahm data to monopoles is an isometry, this means that we can translate results about the geometry of our Nahm moduli spaces into information about time-evolution of $S U(3)$ monopoles. It is known, from the work of Nakajima and Hurtubise $[\mathrm{Na}, \mathrm{Hu} 2]$, that the Nahm transform is an isometry of moduli spaces in the case of $S U(2)$ monopoles.

\section{Nahm's Equations}

In this section we review some material concerning the Nahm equations, the associated moduli spaces, and hyperkähler geometry.

Let $G$ be a compact, connected simple Lie group, and $A$ a connection on a principal $G$-bundle over $\mathbb{R}^{4}$. Let $F_{A}$ denote the curvature of $A$, and $*$ the Hodge star-operator.

Definition 2.1. The self-dual Yang-Mills (SDYM) equations for $A$ are

$$
* F_{A}=F_{A} \text {. }
$$

If we take coordinates $x_{0}, x_{1}, x_{2}, x_{3}$ on $\mathbb{R}^{4}$ and write

$$
A=\sum A_{i} d x_{i}, \quad F_{A}=\sum F_{i j} d x_{i} d x_{j}
$$

where

$$
F_{i j}=\partial_{i} A_{j}-\partial_{j} A_{i}+\left[A_{i}, A_{j}\right]
$$

then the SDYM equations become

$$
\begin{aligned}
& F_{01}=F_{23}, \\
& F_{02}=F_{31}, \\
& F_{03}=F_{12} .
\end{aligned}
$$

If we now impose the condition that the connection components $A_{i}$ are independent of $x_{1}, x_{2}, x_{3}$, and if we relabel $x_{0}$ as $t$, then we arrive at Nahm's equations

$$
\begin{aligned}
& \frac{d A_{1}}{d t}+\left[A_{0}, A_{1}\right]=\left[A_{2}, A_{3}\right], \\
& \frac{d A_{2}}{d t}+\left[A_{0}, A_{2}\right]=\left[A_{3}, A_{1}\right], \\
& \frac{d A_{3}}{d t}+\left[A_{0}, A_{3}\right]=\left[A_{1}, A_{2}\right] .
\end{aligned}
$$

The gauge group of smooth $G$-valued functions on $\mathbb{R}$ acts on solutions to Nahm's equations by

$$
\begin{aligned}
& A_{0} \mapsto g A_{0} g^{-1}-\frac{d g}{d t} g^{-1}, \\
& A_{\imath} \mapsto g A_{i} g^{-1} \quad(i=1,2,3) .
\end{aligned}
$$


The space of solutions to Nahm's equations modulo the action of the gauge group is called the moduli space.

Observe that if we take the connection $A$ to be independent of $x_{0}$ only we arrive at the Bogomolny equations

$$
\begin{aligned}
& \nabla_{1} \Phi=F_{23}, \\
& \nabla_{2} \Phi=F_{31}, \\
& \nabla_{3} \Phi=F_{12},
\end{aligned}
$$

where we denote $-A_{0}$ by $\Phi$ and the covariant derivative $\partial_{i}+\left[A_{i},\right]$ by $\nabla_{i}$.

Solutions $(A, \Phi)$ to the Bogomolny equations such that

$$
\|\Phi\|=a-\frac{b}{r}+O\left(\frac{1}{r^{2}}\right)
$$

for large radial distance $r$ from the origin, where $a$ and $b$ are constants, are known as monopoles. The Higgs field of a monopole, restricted to a sphere of large radius, defines a homotopy class known as the charge. This is a single integer for $S U(2)$ monopoles. data.

There is a remarkable relationship between moduli spaces of monopoles and Nahm

Theorem 2.2. [H1] Consider the space of quadruples $\left(T_{0}, T_{1}, T_{2}, T_{3}\right)$, where

(i) Each $T_{i}$ is a $\mathfrak{u}(k)$-valued function on $[0,2] . T_{0}$ is analytic on $[0,2] ; T_{i}(i=1,2,3)$ is analytic on $(0,2)$ with simple poles at $t=0,2$.

(ii) $T_{0}, T_{1}, T_{2}, T_{3}$ satisfy Nahm's equations.

(iii) The residues of $T_{1}, T_{2}, T_{3}$ at $t=0$ form the irreducible $k$-dimensional representation of $S U(2)$.

(iv) $T_{i}(2-t)=\left(T_{i}(t)\right)^{T}$ for $t \in[0,2]$.

The quotient of this space by the group $\left\{g \in C^{\omega}([0,2], U(k)): g(2-t)=\left(g(t)^{T}\right)^{-1}\right\}$ is naturally equivalent to the moduli space of charge $k S U(2)$-monopoles.

This equivalence between moduli spaces of solutions to reductions of SYDM appears to be a general phenomenon. Another example is the equivalence between moduli spaces for the full SYDM equations and the ADHM equations. The latter may be interpreted as the SYDM equations with invariance under $\mathbb{R}^{4}$ translations imposed. An account of this is given in [CG].

As mentioned in the introduction, our moduli spaces will admit hyperkähler structures. We shall now give some details of this.

Definition 2.3. A hyperkähler structure on a manifold $X$ is a quadruple $(h, I, J, K)$, where

(1) $h$ is a Riemannian metric on $X$.

(2) $I, J, K$ are complex structures, covariant constant with respect to $h$, and $h$ is Hermitian with respect to $I, J, K$.

(3) The complex structures multiply according to the quaternionic relations; $I J=$ $K=-J I$, etc.

A corollary of this definition is that $(h, a I+b J+c K)$ is a Kähler structure on $X$ whenever $a^{2}+b^{2}+c^{2}=1$. Therefore a hyperkähler manifold has a two-sphere of Kähler structures. It is this fact which lies at the heart of the twistor space construction for hyperkähler manifolds explained in [AH]. 
Suppose now that a group $G$ acts on a hyperkähler manifold $X$ preserving $(h, I, J, K)$. The Kähler structures $(h, I),(h, J),(h, K)$ give us Kähler forms $\omega_{1}, \omega_{2}, \omega_{3}$. If $G$ is compact or semisimple there will exist $G$-equivariant moment maps $\mu_{1}, \mu_{2}, \mu_{3}: X \mapsto \mathfrak{g}^{*}$, defined by

$$
\left\langle\mu_{i}(x), \xi\right\rangle=f_{i}^{\xi}(x),
$$

where $d f_{i}^{\xi}$ is the contraction of $\omega_{i}$ with $\xi$. Here we identify an element $\xi \in \mathfrak{g}$ with a vector field on $X$.

We can combine the $\mu_{\imath}$ into a single map $\mu: X \mapsto \mathbb{R}^{3} \otimes \mathfrak{g}^{*}$.

Theorem 2.4. [HKLR] If $q_{1}, q_{2}, q_{3}$ are elements of the centre of $\mathfrak{g}^{*}$, then $\mu^{-1}\left(q_{1}, q_{2}\right.$, $\left.q_{3}\right) / G$ is hyperkähler (when it is a manifold).

This result, known as the hyperkähler quotient construction, has provided many examples of hyperkähler manifolds. In particular, it can be applied to moduli spaces of Nahm data, as the following theorem shows.

Theorem 2.5. [H2] Let $A$ denote the infinite-dimensional quaternionic affine space whose elements are of the form $\frac{d}{d t}+T_{0}+i T_{1}+j T_{2}+k T_{3}$, where $T_{\imath}:[0,2] \mapsto \mathfrak{u}(k)$, $T_{0}$ is analytic on [0,2], $T_{1}, T_{2}, T_{3}$ are analytic on $(0,2)$ with simple poles of residue $\varrho_{i}$, at $t=0$, and $T_{i}(2-t)=\left(T_{i}(t)\right)^{T}$ for $t \in[0,2]$.

Let $\hat{G}=\left\{g \in C^{\omega}[(0,2], U(k)): g(0)=I d, g(2-t)=\left(g(t)^{T}\right)^{-1}\right\}$ act on $\mathcal{A}_{\text {b }}$ by

$$
\begin{aligned}
& T_{0} \mapsto g T_{0} g^{-1}-\frac{d g}{d t} g^{-1}, \\
& T_{i} \mapsto g T_{i} g^{-1} \quad(i=1,2,3) .
\end{aligned}
$$

Define a metric $\tilde{h}$ on $\mathscr{A}$ by

$$
\tilde{h}(Y, Y)=\int_{0}^{2} \sum_{i=0}^{3}\left\langle Y_{\imath}, Y_{i}\right\rangle d t,
$$

where $Y=\left(Y_{0}, Y_{1}, Y_{2}, Y_{3}\right)$.

Define complex structures $\tilde{I}, \tilde{J}, \tilde{K}$ on $\mathscr{b}$ by right multiplication by $-i,-j,-k$ respectively.

Then $(\tilde{h}, \tilde{I}, \tilde{J}, \tilde{K})$ is a hyperkähler structure on $\mathscr{A}$, preserved by the action of $\hat{G}$, and the moment map for the action of $\hat{G}$ is

$$
\left(T_{0}, T_{1}, T_{2}, T_{3}\right) \mapsto\left(\begin{array}{l}
\frac{d T_{1}}{d t}+\left[T_{0}, T_{1}\right]-\left[T_{2}, T_{3}\right] \\
\frac{d T_{2}}{d t}+\left[T_{0}, T_{2}\right]-\left[T_{3}, T_{1}\right] \\
\frac{d T_{3}}{d t}+\left[T_{0}, T_{3}\right]-\left[T_{1}, T_{2}\right]
\end{array}\right) .
$$

Therefore the moduli space of solutions to Nahm's equations with residues $\varrho_{i}$ at $t=0$ is the hyperkähler quotient of $\mathscr{A}$ by $\hat{G}$.

Note that it was necessary to consider Nahm data with fixed residues $\varrho_{i}$ to ensure that the tangent vectors to $\mathscr{C}$ were finite at $t=0,2$ and that the $L^{2}$ metric $\tilde{h}$ was defined. 


\section{The Moduli Space}

We shall now introduce a moduli space $M^{12}$ of Nahm data with modified boundary conditions, and investigate its topology and isometries. We shall be particularly interested in a hyperkähler quotient $M^{8}$ of $M^{12}$ by a circle action.

Definition 3.1. Consider the space $C$ of quadruples $\left(T_{0}, T_{1}, T_{2}, T_{3}\right)$, where

(i) $T_{i}(i=0,1,2,3)$ is a $u(2)$-valued function on $[0,3]$.

(ii) $T_{0}$ is analytic on $[0,3] . T_{1}, T_{2}, T_{3}$ are analytic on $(0,3]$ with simple poles at $t=0$ of residue $-\frac{1}{2} \sigma_{1},-\frac{1}{2} \sigma_{2},-\frac{1}{2} \sigma_{3}$, where $\sigma_{\imath}$ are the Pauli spin matrices.

(iii) The $T_{i}$ satisfy Nahm's equations.

Note the differences with the conditions of 2.2. We now require the Nahm matrices to be analytic at the upper end of the interval on which they are defined, and we have dropped condition (iv) of 2.2 .

In the construction which produces monopoles from Nahm matrices, the interval on which the matrices are defined determines the eigenvalues of the Higgs field at infinity. The choice of $[0,3]$ as interval reflects a choice of these eigenvalues to be $2,-1,-1$, as is discussed in [D1]. Of course, the choice of interval makes no significant difference to the geometry of the moduli spaces.

Let $G_{0}^{0}$ denote the group of analytic $U(2)$-valued functions on $[0,3]$ which are the identity at $t=0,3$. This group acts on $C$ as in 2.5 .

Let $M^{12}$ be the quotient of $C$ by $G_{0}^{0}$.

Theorem 3.2. $M^{12}$ admits a hyperkähler structure.

Proof. This is very similar to the proof of 2.5 . We consider the space $\mathscr{B}$ whose elements are of the form $\frac{d}{d t}+T_{0}+i T_{1}+j T_{2}+k T_{3}$, where $T_{0}, T_{1}, T_{2}, T_{3}$ satisfy (i), (ii) above. The metric $\tilde{h}$ and complex structures $\tilde{I}, \tilde{J}, \tilde{K}$ on $\mathscr{B}$ are defined as in 2.5 , and they define a hyperkähler structure on $\mathscr{B}$ preserved by the action of $G_{0}^{0}$. If we denote by $f$ the induced moment map, then the hyperkähler quotient $f^{-1}(0,0,0) / G_{0}^{0}$ is just $M^{12}$.

Let us now discuss the group actions on $M^{12}$.

(a) There is an action of $\mathbb{R}^{3}$ on $M^{12}$, given by

$$
T_{j} \mapsto T_{j}-i \lambda_{j} I d \quad(j=1,2,3),
$$

where $\left(\lambda_{1}, \lambda_{2}, \lambda_{3}\right) \in \mathbb{R}^{3}$.

(b) $G_{0}=\left\{g \in C^{\omega}([0,3], U(2)): g(0)=I d\right\}$ acts on $f^{-1}(0,0,0) \subset \mathscr{B}$ as in 2.5. This action descends to an action of $G_{0} / G_{0}^{0} \cong U(2)$ on $M^{12}$.

(c) There is an action on $M^{12}$ of $\operatorname{Spin}(3)$, the double cover of $S O(3)$, defined as follows.

Let $P \in \operatorname{Spin}(3)$ descend to $\left(a_{i j}\right) \in S O(3)$. Let $g \in C^{\omega}([0,3], U(2))$ satisfy $g(0)=P, g(3)=I d$. Then the formula

$$
\begin{aligned}
& T_{0} \mapsto g T_{0} g^{-1}-\frac{d g}{d t} g^{-1}, \\
& T_{1} \mapsto g\left(\sum a_{1 j} T_{j}\right) g^{-1}, \\
& T_{2} \mapsto g\left(\sum a_{2 \jmath} T_{j}\right) g^{-1}, \\
& T_{3} \mapsto g\left(\sum a_{3 \jmath} T_{\jmath}\right) g^{-1}
\end{aligned}
$$


defines the Spin(3) action. This is well-defined because any two choices of $g$ differ by an element of $G_{0}^{0}$.

Note that the mapping

$$
\begin{aligned}
& T_{0} \mapsto T_{0}, \\
& T_{i} \mapsto \sum a_{i j} T_{j}
\end{aligned}
$$

preserves Nahm's equations but not the condition on the residues of the Nahm matrices at $t=0$, so does not define an action on $M^{12}$.

Proposition 3.3. The actions of $U(2)$ and $\operatorname{Spin}(3)$ on $M^{12}$ commute.

Proof. It readily follows from the above description of these actions that they commute up to an action of the gauge group $G_{0}^{0}$. As $G_{0}^{0}$ acts trivially on the moduli space $M^{12}$ by definition, the result follows.

Let us now discuss the Riemannian structure of our moduli space. The natural metric on $M^{12}$ is the quotient metric on $f^{-1}(0,0,0) / G_{0}^{0}$, which is defined as follows.

Let $\pi$ denote the projection from $f^{-1}(0,0,0)$ to $M^{12}=f^{-1}(0,0,0) / G_{0}^{0}$. The tangent space to $M^{12}$ at a point $T$ is isomorphic to the subspace of the tangent space to $f^{-1}(0,0,0)$ at $S \in \pi^{-1}(T)$ which is orthogonal to the orbit of $G_{0}^{0}$ through $S$. This is referred to as the space of horizontal tangent vectors at $S$. Given tangent vectors $X, Y$ to $M^{12}$ at $T$, we identify them using the above isomorphism with horizontal tangent vectors $\tilde{X}, \tilde{Y}$ to $f^{-1}(0,0,0)$ at $S$, and define $h(X, Y)=\tilde{h}(\tilde{X}, \tilde{Y})$. This definition is independent of the choice of $S$ in $\pi^{-1}(T)$ because the metric $\tilde{h}$ is $G_{0}^{0}$-invariant.

It follows that the tangent vectors to $M^{12}$ at $\left(T_{0}, T_{1}, T_{2}, T_{3}\right)$ satisfy

(i) The linearisation of Nahm's equations at $\left(T_{0}, T_{1}, T_{2}, T_{3}\right)$.

(ii) Orthogonality to $\left(\frac{d \psi}{d t}+\left[T_{0}, \psi\right],\left[T_{1}, \psi\right],\left[T_{2}, \psi\right],\left[T_{3}, \psi\right]\right)$ for all $\psi \in \operatorname{Lie}\left(G_{0}^{0}\right)$.

The tangent vectors will also be analytic on $[0,3]$, because the residues of the Nahm matrices at $t=0$ are fixed.

This yields the following result.

Proposition 3.4. The tangent space to $M^{12}$ at $\left(T_{0}, T_{1}, T_{2}, T_{3}\right)$ consists of vectors $\left(Y_{0}, Y_{1}, Y_{2}, Y_{3}\right)$ where $Y_{i}:[0,3] \rightarrow \mathfrak{u}(2)$ such that

(i)

$$
\begin{aligned}
\dot{Y}_{1}+\left[Y_{0}, T_{1}\right]+\left[T_{0}, Y_{1}\right] & =\left[T_{2}, Y_{3}\right]+\left[Y_{2}, T_{3}\right], \\
\dot{Y}_{2}+\left[Y_{0}, T_{2}\right]+\left[T_{0}, Y_{2}\right] & =\left[T_{3}, Y_{1}\right]+\left[Y_{3}, T_{1}\right], \\
\dot{Y}_{3}+\left[Y_{0}, T_{3}\right]+\left[T_{0}, Y_{3}\right] & =\left[T_{1}, Y_{2}\right]+\left[Y_{1}, T_{2}\right], \\
\dot{Y}_{0}+\sum_{i=0}^{3}\left[T_{i}, Y_{\imath}\right] & =0 .
\end{aligned}
$$

(ii) $Y_{i}$ is analytic on $[0,3]$ for $i=0,1,2,3$.

Proposition 3.5. Let

$$
\left(\begin{array}{lll}
a_{1} & a_{2} & a_{3} \\
b_{1} & b_{2} & b_{3} \\
c_{1} & c_{2} & c_{3}
\end{array}\right)
$$

be an element of $S O(3)$. Let $g$ be an element of $C^{\omega}([0,3], U(2))$. Then if $\left(Y_{0}, Y_{1}, Y_{2}, Y_{3}\right)$ satisfies Eq. (11-14) at $\left(T_{0}, T_{1}, T_{2}, T_{3}\right)$ then $\left(g Y_{0} g^{-1}, g\left(\sum a_{i} Y_{i}\right) g^{-1}, g\left(\sum b_{i} Y_{i}\right) g^{-1}\right.$, 
$\left.g\left(\sum c_{\imath} Y_{i}\right) g^{-1}\right)$ satisfies $(11-14)$ at $\left(g T_{0} g^{-1}-\dot{g} g^{-1}, g\left(\sum a_{i} T_{\imath}\right) g^{-1}, g\left(\sum b_{\imath} T_{i}\right) g^{-1}\right.$, $\left.g\left(\sum c_{i} T_{\imath}\right) g^{-1}\right)$.

Proof. This is a straightforward calculation.

This result means that to find the tangent space at an arbitrary point of $M^{12}$, we need only compute the tangent space at one point in each $U(2) \times \operatorname{Spin}(3)$ orbit on $M^{12}$. We shall use this in Sect. 4, when we calculate metrics on moduli spaces.

Differentiation of the $\mathbb{R}^{3}, U(2)$ and $\operatorname{Spin}(3)$ actions yields

Proposition 3.6. $\mathbb{R}^{3}$ and $U(2) \times \operatorname{Spin}(3)$ act isometrically on $M^{12}$.

The Kähler forms on $M^{12}$ associated to the complex structures $I, J, K$ are defined as follows. We have Kähler forms $\tilde{\omega}_{1}, \tilde{\omega}_{2}, \tilde{\omega}_{3}$ on . $\mathcal{P}$ defined by

$$
\tilde{\omega}_{1}(\tilde{X}, \tilde{Y})=\tilde{h}(\tilde{I} \tilde{X}, \tilde{Y})
$$

etc. Explicitly

$$
\begin{aligned}
& \tilde{\omega}_{1}\left(\left(\tilde{X}_{0}, \tilde{X}_{1}, \tilde{X}_{2}, \tilde{X}_{3}\right),\left(\tilde{Y}_{0}, \tilde{Y}_{1}, \tilde{Y}_{2}, \tilde{Y}_{3}\right)\right) \\
& \quad=\int_{0}^{3}\left\langle\tilde{X}_{0}, \tilde{Y}_{1}\right\rangle-\left\langle\tilde{X}_{1}, \tilde{Y}_{0}\right\rangle+\left\langle\tilde{X}_{3}, \tilde{Y}_{2}\right\rangle-\left\langle\tilde{X}_{2}, \tilde{Y}_{3}\right\rangle d t .
\end{aligned}
$$

Given tangent vectors $X, Y$ on $M^{12}$, lift them to $\tilde{X}, \tilde{Y}$ on $f^{-1}(0,0,0)$ and let $\omega_{\imath}(X, Y)=\tilde{\omega}_{\imath}(\tilde{X}, \tilde{Y})$.

This definition is independent of the lifts chosen because the Kähler forms on $f^{-1}(0,0,0)$ are degenerate along the orbits of the gauge group $G_{0}^{0}$.

It is clear that the actions of $\mathbb{R}^{3}$ and $U(2)$ are triholomorphic, that is, they fix the Kähler structures of $M^{12}$. The Spin(3) action, on the other hand, acts transitively on the two-sphere of Kähler structures. $M^{12}$

We are now in a position to calculate moment maps for triholomorphic actions on

Consider the centre of $U(2)$, that is, the $U(1)$ subgroup of scalar matrices. The elements of this group may be represented by maps

$$
\Psi(\theta): t \mapsto e^{\imath \theta t / 3} I d .
$$

Therefore the image of $\left(T_{0}, T_{1}, T_{2}, T_{3}\right)$ under the action of $\Psi(\theta)$ is $\left(T_{0}-\frac{1}{3} i \theta I d\right.$. $\left.T_{1}, T_{2}, T_{3}\right)$.

Differentiating at $\theta=0$ shows that the vector field generated by the $U(1)$ action is constant and its value is $\left(-\frac{1}{3} i I d, 0,0,0\right)$.

We deduce the moment map for this action.

Proposition 3.7. The hyperkähler moment map induced by the action on $M^{12}$ of the centre of $U(2)$ is

$$
m:\left(T_{0}, T_{1}, T_{2}, T_{3}\right) \mapsto 2 i\left(\operatorname{trace} T_{1}, \operatorname{trace} T_{2}, \operatorname{trace} T_{3}\right) .
$$

Note that this is invariant under the action of $G_{0}^{0}$ and so is well-defined on $M^{12}$.

We can now make the following definition.

Definition 3.8. Let $M^{8}$ be the hyperkähler quotient $m^{-1}(0,0,0) / U(1)$. 
We see that $M^{8}$ inherits an $S U(2) \times S O(3)$ action (rather than $\left.S U(2) \times \operatorname{Spin}(3)\right)$ from $M^{12}$. The centre of $S U(2)$ acts trivially. The $S U(2)$ action fixed the complex structures and the $S O(3)$ action rotates them.

Note that $M^{8}$ can also be obtained as the ordinary quotient of $M^{12}$ by $\mathbb{R}^{3} \times U(1)$, where the action of $\mathbb{R}^{3}$ is that defined in (10).

Definition 3.9. Let $N^{5}$ denote the quotient of $M^{8}$ by $S U(2)$.

The $S O(3)$ action descends to the quotient space $N^{5}$ because it commutes with the action of $S U(2)$ on $M^{8}$.

We shall now solve the Nahm equations subject to the conditions of 3.1, and hence obtain a concrete description of our moduli spaces $M^{12}, M^{8}$ and $N^{5}$.

Let us first factor out the action of $\mathbb{R}^{3}$ and the centre of $U(2)$. This just amounts to making the Nahm matrices $T_{0}, T_{1}, T_{2}, T_{3}$ tracefree. We can now use the $S U(2)$ action to gauge $T_{0}$ to zero; Nahm's equations now become

$$
\begin{aligned}
& \dot{T}_{1}=\left[T_{2}, T_{3}\right], \\
& \dot{T}_{2}=\left[T_{3}, T_{1}\right], \\
& \dot{T}_{3}=\left[T_{1}, T_{2}\right] .
\end{aligned}
$$

The space of solutions to these equations which satisfy our boundary conditions is the space $N^{5}$ of Definition 3.9. It follows from (18-20) that the quantities

$$
\begin{aligned}
& \alpha_{1}=\left\langle T_{1}, T_{1}\right\rangle-\left\langle T_{2}, T_{2}\right\rangle, \\
& \alpha_{2}=\left\langle T_{1}, T_{1}\right\rangle-\left\langle T_{3}, T_{3}\right\rangle, \\
& \alpha_{3}=\left\langle T_{1}, T_{2}\right\rangle, \\
& \alpha_{4}=\left\langle T_{1}, T_{3}\right\rangle, \\
& \alpha_{5}=\left\langle T_{2}, T_{3}\right\rangle
\end{aligned}
$$

are constant in $t$. In fact, the $\alpha_{i}$ are coordinates on $N^{5}$ and the map

$$
\left(\alpha_{1}, \alpha_{2}, \alpha_{3}, \alpha_{4}, \alpha_{5}\right) \mapsto\left(\begin{array}{ccc}
\frac{1}{3}\left(\alpha_{1}+\alpha_{2}\right) & \alpha_{3} & \alpha_{4} \\
\alpha_{3} & \frac{1}{3}\left(\alpha_{2}-2 \alpha_{1}\right) & \alpha_{5} \\
\alpha_{4} & \alpha_{5} & \frac{1}{3}\left(\alpha_{1}-2 \alpha_{2}\right)
\end{array}\right)
$$

is an $S O(3)$-equivariant homeomorphism from $N^{5}$ onto an open subset of the space $W_{2}$ of real traceless symmetric $3 \times 3$ matrices. The latter space is a realisation of the irreducible 5-dimensional representation of $S O(3)$. In this case $S O(3)$ acts by conjugation.

Now, any element of $W_{2}$ is conjugate under $S O(3)$ to a diagonal matrix with the eigenvalues arranged in increasing order. From (21) we deduce that every orbit of $S O(3)$ in $N^{5}$ contains a unique point with $\left\langle T_{1}, T_{2}\right\rangle=\left\langle T_{1}, T_{3}\right\rangle=\left\langle T_{2}, T_{3}\right\rangle=0$ and $\left\langle T_{1}, T_{1}\right\rangle \leq\left\langle T_{2}, T_{2}\right\rangle \leq\left\langle T_{3}, T_{3}\right\rangle$. Together with our condition on the residues of the Nahm matrices, this implies that

$$
T_{0}=0, \quad T_{i}=\frac{1}{2} f_{i} \sigma_{i} \quad(i=1,2,3),
$$

where the $f_{i}$ are real-valued functions satisfying

$$
\begin{aligned}
& \dot{f}_{1}=f_{2} f_{3}, \\
& \dot{f}_{2}=f_{3} f_{1}, \\
& \dot{f}_{3}=f_{1} f_{2},
\end{aligned}
$$


and

$$
f_{1}^{2} \leq f_{2}^{2} \leq f_{3}^{2}
$$

The functions $f_{1}, f_{2}, f_{3}$ are analytic on $(0,3]$ and have simple poles at $t=0$ of residue -1 .

The space of solutions to (23-25) satisfying these conditions is in bijective correspondence with the quotient $N^{5} / S O(3)$.

We shall refer to Nahm data of the form (22) as being in diagonal form, because such data corresponds under the mapping (21) to diagonal matrices in $W_{2}$.

Note that $f_{3}^{2}-f_{2}^{2}$ and $f_{3}^{2}-f_{1}^{2}$ are constant in $t$. This enables us to integrate the equations completely. The solution (subject to our conditions) is

$$
\begin{aligned}
& f_{1}(t)=-\frac{D c n_{k}(D t)}{s n_{k}(D t)}, \\
& f_{2}(t)=-\frac{D d n_{k}(D t)}{s n_{k}(D t)}, \\
& f_{3}(t)=-\frac{D}{s n_{k}(D t)},
\end{aligned}
$$

where $0 \leq k \leq 1$ and $D<\frac{2}{3} K(k)$. Here $s n_{k}, c n_{k}$ and $d n_{k}$ are the elliptic functions of Jacobi, and $4 K(k)$ is the period of $s n_{k}$ (see [WW] for details of the Jacobi functions). We need the condition $D<\frac{2}{3} K(k)$ because we require the Nahm matrices to be analytic on $(0,3]$.

We have established the following result.

Proposition 3.10. The quotient space $N^{5} / S O(3)$ may be identified with the set $\left\{(D, k): 0 \leq k \leq 1,0 \leq D<\frac{2}{3} K(k)\right\}$.

Note that this is not a manifold. The reason for this is that the isotropy subgroup for the action of $S O(3)$ is not the same at all points of $N^{5}$.

(i) The point represented by $D=k=0$ is fixed by $S O(3)$. It represents the solution

$$
f_{1}=f_{2}=f_{3}=-\frac{1}{t} \text {. }
$$

We call this the spherically symmetric solution.

(ii) The points given by $k=0$ or $k=1$ have isotropy group $S^{1} \times \mathbf{Z}_{2}$. They represent the solutions

$$
\begin{aligned}
f_{1} & =-D \cot (D t), \\
f_{2}=f_{3} & =-D \operatorname{cosec}(D t),
\end{aligned}
$$

and

$$
\begin{aligned}
f_{1}=f_{2} & =-D \operatorname{cosech}(D t), \\
f_{3} & =-D \operatorname{coth}(D t),
\end{aligned}
$$

respectively.

For obvious reasons we call these trigonometric axisymmetric and hyperbolic axisymmetric solutions respectively.

(iii) Other solutions have a $\mathbf{Z}_{2} \times \mathbf{Z}_{2}$ isotropy group. 
Note that the above statements are about isotropy subgroups of the $S O(3)$ action on $N^{5}=M^{8} / S U(2)$. On the larger space $M^{8}$, the action of $S O(3)$ is free.

We observe that the above results show that every element of $M^{8}$ lies in the same $S U(2) \times S O(3)$ orbit as a point of the form

$$
\left(T_{0}, T_{1}, T_{2}, T_{3}\right)=\left(0, \frac{1}{2} f_{1} \sigma_{1}, \frac{1}{2} f_{2} \sigma_{2}, \frac{1}{2} f_{3} \sigma_{3}\right),
$$

that is, a point in diagonal form.

We conclude this section with some remarks on the topology of our moduli spaces.

Theorem 3.11. The moduli space $N^{5}$ is homeomorphic to $\mathbb{R}^{5}$.

Proof. It follows as in [Hu1] that $N^{5}$ is homeomorphic to $\left\{a \in W_{2}: \operatorname{Tr} a^{2}<1\right\}$, the open unit ball in $W_{2} \cong \mathbb{R}^{5}$.

It is easy to check that the actions of $U(2)$ and $\mathbb{R}^{3}$ on $M^{12}$, and the action of $S U(2) / \mathbf{Z}_{2}$ on $M^{8}$, are free. We deduce the next result.

Corollary 3.12. $M^{8}$ is homeomorphic to $\mathbb{R}^{5} \times S U(2) / \mathbf{Z}_{2}$, and $M^{12}$ is homeomorphic to $\mathbb{R}^{8} \times U(2)$.

\section{The Metric}

In general the problem of calculating the metric on a moduli space is a difficult one. In our case, however, we are helped by the explicit formulae we have for the solutions of Nahm's equations which represent points of $M^{8}$. We shall explicitly calculate the tangent space to $M^{8}$, and use this description to derive an expression for the metric.

Recall from 3.5 that to determine the tangent space at arbitrary points of $M^{8}$ we need only calculate it at one point in each $S U(2) \times S O(3)$ orbit. From the results of Sect. 3, we see that it is enough to find the tangent space at points of $M^{8}$ represented by Nahm data of the form $\left(0, \frac{1}{2} f_{1} \sigma_{1}, \frac{1}{2} f_{2} \sigma_{2}, \frac{1}{2} f_{3} \sigma_{3}\right)$.

Let us write a tangent vector to $M^{8}$ at a point of this form as $\left(Y_{0}, Y_{1}, Y_{2}, Y_{3}\right)$. Using the isomorphism $\mathfrak{s u}(2) \cong \mathbb{R}^{3}$ we may write the components, $Y_{\imath}$ as

$$
\begin{aligned}
& Y_{0}=\left(\begin{array}{l}
w_{1} \\
w_{2} \\
w_{3}
\end{array}\right), \\
& Y_{1}=\left(\begin{array}{l}
x_{1} \\
x_{2} \\
x_{3}
\end{array}\right), \\
& Y_{2}=\left(\begin{array}{l}
y_{1} \\
y_{2} \\
y_{3}
\end{array}\right), \\
& Y_{3}=\left(\begin{array}{l}
z_{1} \\
z_{2} \\
z_{3}
\end{array}\right) .
\end{aligned}
$$

The equations (11-14) that $Y_{i}$ must satisfy to be a tangent vector are

$$
\begin{aligned}
& \dot{x}_{1}=f_{2} z_{3}+f_{3} y_{2}, \\
& \dot{y}_{2}=f_{3} x_{1}+f_{1} z_{3}, \\
& \dot{z}_{3}=f_{1} y_{2}+f_{2} x_{1},
\end{aligned}
$$


and three similar systems, each of three equations in three variables. These equations may be solved exactly by elementary methods, and we arrive at the following theorem.

Theorem 4.1. The tangent space to $M^{8}$ at $\left(0, \frac{1}{2} f_{1} \sigma_{1}, \frac{1}{2} f_{2} \sigma_{2}, \frac{1}{2} f_{3} \sigma_{3}\right)$ consists of vectors $\left(Y_{0}, Y_{1}, Y_{2}, Y_{3}\right)$, where

$$
\begin{aligned}
& Y_{0}=\left(\begin{array}{c}
\dot{f}_{1} I_{4} \\
\dot{f}_{2} I_{3}+\frac{m_{3}}{f_{2}} \\
-\dot{f}_{3} I_{2}-\frac{n_{2}}{f_{3}}
\end{array}\right), \\
& Y_{1}=\left(\begin{array}{c}
\dot{f}_{1} I_{1} \\
\dot{f}_{2} I_{2}+\frac{m_{2}}{f_{2}} \\
\dot{f}_{3} I_{3}+\frac{n_{3}}{f_{3}}
\end{array}\right), \\
& Y_{2}=\left(\begin{array}{c}
-\dot{f}_{1} I_{2} \\
\dot{f}_{2} I_{1}+\frac{m_{1}}{f_{2}} \\
-\dot{f}_{3} I_{4}-\frac{n_{4}}{f_{3}}
\end{array}\right) \text {, } \\
& Y_{3}=\left(\begin{array}{c}
-\dot{f}_{1} I_{3} \\
\dot{f}_{2} I_{4}+\frac{m_{4}}{f_{2}} \\
\dot{f}_{3} I_{1}+\frac{n_{1}}{f_{3}}
\end{array}\right),
\end{aligned}
$$

and

$$
I_{\jmath}=\int_{0}^{t} \frac{m_{\jmath}}{f_{2}^{2}(s)}+\frac{n_{j}}{f_{3}^{2}(s)} d s
$$

for $j=1,2,3,4$.

The parameters $m_{i}, n_{\imath}(i=1,2,3,4)$ are coordinates for the tangent space.

We are now in a position to make some statements about the Riemannian geometry of $M^{8}$.

Theorem 4.2. $M^{8}$ is irreducible as a Riemannian manifold.

Proof. $M^{8}$ is a hyperkähler 8-manifold, so if it is reducible it must be a product $P \times Q$, where $P, Q$ are hyperkähler 4-manifolds.

Let $p$ denote the point of $M^{8}$ represented by $\left(0,-\frac{1}{2 t} \sigma_{1},-\frac{1}{2 t} \sigma_{2},-\frac{1}{2 t} \sigma_{3}\right)$. This is the spherically symmetric solution (30). Now the diagonal subgroup $S O(3)$ of the isometry group $S U(2) / \mathbf{Z}_{2} \times S O(3)$ fixes $p$, so the tangent space to $M^{8}$ at $p$ is an 8-dimensional representation of $S O(3)$. Using the explicit description given by 4.1 it can be checked that the tangent space is isomorphic as an $S O(3)$-module to $W_{1} \oplus W_{2}$, where $W_{n}$ denotes the irreducible $(2 n+1)$-dimensional representation of $S O(3)$.

If we have a decomposition $M^{8}=P \times Q$, however, then the tangent space is a direct sum of two 4-dimensional representations of $S O(3)$, giving a contradiction. 
The presence of a large group of isometries of $M^{8}$ gives us a supply of totally geodesic submanifolds.

Proposition 4.3. Let $\Gamma$ denote the curve in $M^{8}$ represented by Nahm data of the form $\left(0, \frac{1}{2} f_{1} \sigma_{1}, \frac{1}{2} f_{2} \sigma_{2}, \frac{1}{2} f_{3} \sigma_{3}\right)$, where $f_{2}^{2}=f_{3}^{2}$. Let $\Sigma$ denote the circle bundle over $\Gamma$ obtained by applying the action of the $U(1)$ subgroup of $S U(2)$ whose elements are of the form

$$
g=\left(\begin{array}{cc}
e^{\imath \theta} & 0 \\
0 & e^{-i \theta}
\end{array}\right)
$$

Then the surface $\Sigma$ is totally geodesic in $M^{8}$. Moreover the image of $\Gamma$ in $N^{5}$ under the projection $M^{8} \rightarrow N^{5}=M^{8} / S U(2)$ is a geodesic in $N^{5}$.

Proof. $\Sigma$ is the fixed point set of the diagonal subgroup of the isometry group $S U(2) \times S O(3)$ given by

$$
\left\{\left(\begin{array}{cc}
e^{i \theta / 2} & 0 \\
0 & e^{-i \theta / 2}
\end{array}\right),\left(\begin{array}{ccc}
1 & 0 & 0 \\
0 & \cos \theta & \sin \theta \\
0 & -\sin \theta & \cos \theta
\end{array}\right): \theta \in[0,2 \pi]\right\} .
$$

The image of $\Gamma$ is the fixed point set in $N^{5}$ of the circle subgroup of $S O(3)$

$$
\left\{\left(\begin{array}{ccc}
1 & 0 & 0 \\
0 & \cos \theta & \sin \theta \\
0 & -\sin \theta & \cos \theta
\end{array}\right): \theta \in[0,2 \pi]\right\} \text {. }
$$

Both these groups act isometrically, so the result follows.

We can perform a similar construction by taking other circle subgroups of $S O(3)$ and considering the fixed point sets of the associated diagonal subgroup of the isometry group. In this way we obtain a totally geodesic surface in $M^{8}$ for each point in $\mathbb{R P}^{2}$. We can view these surfaces as representing Nahm data axisymmetric about an axis through the origin in $\mathbb{R}^{3}$.

We have been unable to show the existence of any compact totally geodesic submanifolds of $M^{8}$. However we do have the following weaker result.

Proposition 4.4. Let $\Omega$ denote the $\mathbb{R P}^{3}$ of spherically symmetric Nahm data in $M^{8}$; that is, the orbit of the isometry group of $M^{8}$ containing the point $p$ defined in 4.2 . Then $\Omega$ is a minimal submanifold of $M^{8}$.

Proof. We have to show that the second fundamental form $B$ of the embedding $\Omega \hookrightarrow M^{8}$ is tracefree.

Now, if $\mathscr{T}$ denotes the tangent space to $\Omega$ at $p$ and $\mathscr{N}^{\circ}$ denotes the normal space, then $B$ lies in $S^{2}(\mathscr{T}) \otimes \mathscr{N}$. Observing that the tangent space to $M^{8}$ at $p$ is the direct sum of $\mathscr{T}$ and $\mathscr{N}$, and using the results and notation of 4.2 , we see that $\mathscr{T} \cong W_{1}$ and $\mathscr{N} \cong W_{2}$ as $S O(3)$-modules. Using the Clebsch-Gordan formula it follows that: $S^{2}(\mathscr{T}) \otimes \mathscr{N}^{2} \cong\left(W_{2} \oplus W_{0}\right) \otimes W_{2}$, where the first $W_{2}$ represents the tracefree tensors in $S^{2}(\mathscr{T})$. By Schur's Lemma, $B$ is tracefree at $p$.

The same argument works (using a different $S O(3)$ ), for each point of $\Omega$.

Let us now calculate the metrics on our moduli spaces. First we shall consider the quotient metric on the 5-manifold $N^{5}=M^{8} / S U(2)$. 
Let us take as coordinates on $N^{5}$ the quantities $\alpha_{1}, \ldots, \alpha_{5}$ defined earlier by

$$
\begin{aligned}
& \alpha_{1}=\left\langle T_{1}, T_{1}\right\rangle-\left\langle T_{2}, T_{2}\right\rangle, \\
& \alpha_{2}=\left\langle T_{1}, T_{1}\right\rangle-\left\langle T_{3}, T_{3}\right\rangle, \\
& \alpha_{3}=\left\langle T_{1}, T_{2}\right\rangle, \\
& \alpha_{4}=\left\langle T_{1}, T_{3}\right\rangle, \\
& \alpha_{5}=\left\langle T_{2}, T_{3}\right\rangle .
\end{aligned}
$$

These are $S U(2)$ and gauge-invariant, and independent of $t$ as a consequence of Nahm's equations.

We shall calculate tangent vectors $\tilde{Y}\left(\alpha_{i}\right)$ which are horizontal (i.e. orthogonal to the $S U(2)$ orbits in $M^{8}$ ) and dual to the above coordinates. The latter condition may be expressed as

$$
d \alpha_{i}\left(\tilde{Y}\left(\alpha_{j}\right)\right)=\delta_{i \jmath} .
$$

The inner products of these tangent vectors will give us the terms of the metric. Explicitly, the metric will be

$$
d s^{2}=\sum_{i, \jmath=1}^{5} G_{\imath \jmath} d \alpha_{i} d \alpha_{j}
$$

where $G_{i j}=\int_{0}^{3}\left\langle\tilde{Y}\left(\alpha_{\imath}\right), \tilde{Y}\left(\alpha_{\jmath}\right)\right\rangle d t$.

It will be useful to establish the following lemma.

Lemma 4.5. Let $Y, Z$ be tangent vectors to $M^{8}$ at $\left(0, \frac{1}{2} f_{1} \sigma_{1}, \frac{1}{2} f_{2} \sigma_{2}, \frac{1}{2}, f_{3} \sigma_{3}\right)$, given, in the notation of 4.1 , by parameters $m_{i}, n_{i}$, and $m_{\imath}^{\prime}, n_{i}^{\prime}$ respectively.

Then their inner product is given by

$$
\langle Y, Z\rangle=\sum_{i=1}^{4} m_{i} m_{i}^{\prime}\left(h_{1}+g_{1}\right)+n_{i} n_{\imath}^{\prime}\left(h_{3}+g_{2}\right)+\left(m_{\imath} n_{i}^{\prime}+m_{i}^{\prime} n_{i}\right) h_{2},
$$

where

$$
\begin{aligned}
& h_{1}=f_{1}(3) f_{2}(3) f_{3}(3)\left(\int_{0}^{3} \frac{1}{f_{2}^{2}}\right)^{2}, \\
& h_{2}=f_{1}(3) f_{2}(3) f_{3}(3)\left(\int_{0}^{3} \frac{1}{f_{2}^{2}}\right)\left(\int_{0}^{3} \frac{1}{f_{3}^{2}}\right), \\
& h_{3}=f_{1}(3) f_{2}(3) f_{3}(3)\left(\int_{0}^{3} \frac{1}{f_{3}^{2}}\right)^{2}, \\
& g_{1}=\int_{0}^{3} \frac{1}{f_{2}^{2}}, \\
& g_{2}=\int_{0}^{3} \frac{1}{f_{3}^{2}} .
\end{aligned}
$$


Proof. This follows from direct calculation, using integration by parts and the relation

$$
\left(\dot{f}_{1}\right)^{2}+\left(\dot{f}_{2}\right)^{2}+\left(\dot{f}_{3}\right)^{2}=\frac{d}{d t}\left(f_{1} f_{2} f_{3}\right)
$$

Now, an arbitrary point of $N^{5}=M^{8} / S U(2)$ is of the form $\left(\tilde{T}_{0}, \tilde{T}_{1}, \tilde{T}_{2}, \tilde{T}_{3}\right)=$ $\left(-\dot{A}(t) A(t)^{-1}, A(t) \sum a_{\imath} T_{\imath} A(t)^{-1}, A(t) \sum b_{i} T_{i} A(t)^{-1}, A(t) \sum c_{i} T_{i} A(t)^{-1}\right)$, where $T_{i}=\frac{1}{2} f_{i} \sigma_{i}(i=1,2,3)$

$$
A=\left(\begin{array}{lll}
a_{1} & a_{2} & a_{3} \\
b_{1} & b_{2} & b_{3} \\
c_{1} & c_{2} & c_{3}
\end{array}\right) \in S O(3)
$$

and $t \mapsto A(t)$ is an analytic map from $[0,3]$ to $S U(2)$ with $A(3)=I d$ and $A(0)$ descending to $A$ under the double cover $S U(2) \mapsto S O(3)$.

Equivalently, $\left(\tilde{T}_{0}, \tilde{T}_{1}, \tilde{T}_{2}, \tilde{T}_{3}\right)$ is obtained by applying the $S O(3)$ action of $A$ to a point in diagonal form.

The tangent vectors to $M^{8}$ at $\left(\tilde{T}_{0}, \tilde{T}_{1}, \tilde{T}_{2}, \tilde{T}_{3}\right)$ are of the form:

$$
\begin{aligned}
\tilde{Y}= & \left(\tilde{Y}_{0}, \tilde{Y}_{1}, \tilde{Y}_{2}, \tilde{Y}_{3}\right) \\
= & \left(A(t) Y_{0} A(t)^{-1}, A(t) \sum a_{i} Y_{i} A(t)^{-1}, A(t) \sum b_{i} Y_{i} A(t)^{-1},\right. \\
& \left.A(t) \sum c_{\imath} Y_{\imath} A(t)^{-1}\right)
\end{aligned}
$$

where $Y=\left(Y_{0}, Y_{1}, Y_{2}, Y_{3}\right)$ is a tangent vector to $M^{8}$ at $\left(T_{0}, T_{1}, T_{2}, T_{3}\right)$.

Using the expressions (41-45) for $\alpha_{1}, \ldots, \alpha_{5}$ we find that

$$
\left(\begin{array}{c}
d \alpha_{1}(\tilde{Y}) \\
d \alpha_{2}(\tilde{Y}) \\
d \alpha_{3}(\tilde{Y}) \\
d \alpha_{4}(\tilde{Y}) \\
d \alpha_{5}(\tilde{Y})
\end{array}\right)=R(A)\left(\begin{array}{c}
-2 m_{1} \\
-2 n_{1} \\
m_{2} \\
n_{3} \\
m_{4}-n_{4}
\end{array}\right)
$$

where the matrix $R(A)$ is

$$
\left(\begin{array}{ccccc}
b_{2}^{2}-a_{2}^{2} & b_{3}^{2}-a_{3}^{2} & 2\left(a_{1} a_{2}-b_{1} b_{2}\right) & 2\left(a_{1} a_{3}-b_{1} b_{3}\right) & 2\left(a_{2} a_{3}-b_{2} b_{3}\right) \\
c_{2}^{2}-a_{2}^{2} & c_{3}^{2}-a_{3}^{2} & 2\left(a_{1} a_{2}-c_{1} c_{2}\right) & 2\left(a_{1} a_{3}-c_{1} c_{3}\right) & 2\left(a_{2} a_{3}-c_{2} c_{3}\right) \\
-a_{2} b_{2} & -a_{3} b_{3} & a_{1} b_{2}+a_{2} b_{1} & a_{1} b_{3}+a_{3} b_{1} & a_{2} b_{3}+a_{3} b_{2} \\
-a_{2} c_{2} & -a_{3} c_{3} & a_{1} c_{2}+a_{2} c_{1} & a_{1} c_{3}+a_{3} c_{1} & a_{2} c_{3}+a_{3} c_{2} \\
-b_{2} c_{2} & -b_{3} c_{3} & b_{1} c_{2}+b_{2} c_{1} & b_{1} c_{3}+b_{3} c_{1} & b_{2} c_{3}+b_{3} c_{2}
\end{array}\right)
$$

The mapping $A \mapsto R(A)$ is in fact the irreducible 5-dimensional representation of $S O(3)$, as can be seen by computing its character. Note that $R\left(A^{T}\right)=R(A)^{-1}$ so we can find the inverse of $R(A)$.

The duality relations for $\tilde{Y}\left(\alpha_{1}\right)$ are, therefore

$$
\left(\begin{array}{c}
-2 m_{1} \\
-2 n_{1} \\
m_{2} \\
n_{3} \\
m_{4}-n_{4}
\end{array}\right)=R\left(A^{T}\right)\left(\begin{array}{l}
1 \\
0 \\
0 \\
0 \\
0
\end{array}\right)=R\left(A^{T}\right) e_{1}
$$


where we denote by $e_{j}$ the 5-element column vector with $j^{\text {th }}$ entry equal to 1 and all other entries equal to zero.

We get the duality relations for $\tilde{Y}\left(\alpha_{2}\right), \ldots, \tilde{Y}\left(\alpha_{5}\right)$ by replacing $e_{1}$ by $e_{2}, \ldots, e_{5}$ respectively.

The coordinates $\alpha_{i}$ are invariant under the $S U(2)$ action, so the vertical tangent vectors $Z$ (i.e. the vectors tangent to the $S U(2)$ orbits) are precisely those satisfying $d \alpha_{\imath}(Z)=0(i=1, \ldots, 5)$. Using (54) we can identify the vertical tangent vectors; by applying the result of 4.5 we see that a tangent vector $\tilde{Y}$ is horizontal (i.e. orthogonal to the vertical tangent vectors) if and only if

$$
\begin{aligned}
m_{2} h_{2}+n_{2}\left(h_{3}+g_{2}\right) & =0, \\
m_{3}\left(h_{1}+g_{1}\right)+n_{3} h_{2} & =0, \\
m_{4}\left(h_{1}+h_{2}+g_{1}\right)+n_{4}\left(h_{2}+h_{3}+g_{2}\right) & =0 .
\end{aligned}
$$

We have expressed the duality and horizontality relations that the tangent vectors $\tilde{Y}\left(\alpha_{i}\right)$ must satisfy as a system of linear equations in $m_{i}, n_{i}$. We can now solve these equations and, using Lemma 4.5, read off the inner products that give the terms of the metric.

Theorem 4.6. Let $\left(\tilde{T}_{0}, \tilde{T}_{1}, \tilde{T}_{2}, \tilde{T}_{3}\right)$ be the image of $\left(0, \frac{1}{2} f_{1} \sigma_{1}, \frac{1}{2} f_{2} \sigma_{2}, \frac{1}{2} f_{3} \sigma_{3}\right)$ under the action of $A=\left(\begin{array}{lll}a_{1} & a_{2} & a_{3} \\ b_{1} & b_{2} & b_{3} \\ c_{1} & c_{2} & c_{3}\end{array}\right) \in S O(3)$.

Then the metric of $N^{5}$ at that point is

$$
d s^{2}=\sum_{i, j=1}^{5} G_{i j} d \alpha_{i} d \alpha_{j}
$$

where

$$
G_{i j}=\sum_{k=1}^{4} m_{k}^{(i)} m_{k}^{(j)}\left(h_{1}+g_{1}\right)+n_{k}^{(i)} n_{k}^{(j)}\left(h_{3}+g_{2}\right)+\left(m_{k}^{(i)} n_{k}^{(j)}+m_{k}^{(j)} n_{k}^{(i)}\right) h_{2},
$$

$g_{1}, g_{2}, h_{1}, h_{2}, h_{3}$ are defined as in Lemma 4.5, and $m_{k}^{(i)}, n_{k}^{(i)}(k=1,2,3,4)$ are given by the following formulae.

(i)

$$
\begin{aligned}
& m_{1}^{(1)}=\frac{1}{2}\left(b_{1}^{2}-b_{2}^{2}\right) \\
& m_{2}^{(1)}=-b_{1} b_{2} \\
& m_{3}^{(1)}=\frac{b_{1} b_{3} h_{2}}{h_{1}+g_{1}} \\
& m_{4}^{(1)}=\frac{-b_{2} b_{3}\left(h_{2}+h_{3}+g_{2}\right)}{h_{1}+2 h_{2}+h_{3}+g_{1}+g_{2}}, \\
& n_{1}^{(1)}=\frac{1}{2}\left(b_{1}^{2}-b_{3}^{2}\right) \\
& n_{2}^{(1)}=\frac{b_{1} b_{2} h_{2}}{h_{3}+g_{2}} \\
& n_{3}^{(1)}=-b_{1} b_{3} \\
& n_{4}^{(1)}=\frac{b_{2} b_{3}\left(h_{1}+h_{2}+g_{1}\right)}{h_{1}+2 h_{2}+h_{3}+g_{1}+g_{2}} .
\end{aligned}
$$


(ii) The expressions for $m_{i}^{(2)}, n_{i}^{(2)}$ are the same as those above except that $b_{j}$ is replaced by $c_{j}$ throughout.

(iii)

$$
\begin{aligned}
& m_{1}^{(3)}=a_{2} b_{2}-a_{1} b_{1} \\
& m_{2}^{(3)}=a_{1} b_{2}+a_{2} b_{1} \\
& m_{3}^{(3)}=-\frac{\left(a_{1} b_{3}+a_{3} b_{1}\right) h_{2}}{h_{1}+g_{1}} \\
& m_{4}^{(3)}=\frac{\left(a_{2} b_{3}+a_{3} b_{2}\right)\left(h_{2}+h_{3}+g_{2}\right)}{h_{1}+2 h_{2}+h_{3}+g_{1}+g_{2}} \\
& n_{1}^{(3)}=a_{3} b_{3}-a_{1} b_{1} \\
& n_{2}^{(3)}=-\frac{\left(a_{1} b_{2}+a_{2} b_{1}\right) h_{2}}{h_{3}+g_{2}} \\
& n_{3}^{(3)}=a_{1} b_{3}+a_{3} b_{1}, \\
& n_{4}^{(3)}=-\frac{\left(a_{2} b_{3}+a_{3} b_{2}\right)\left(h_{1}+h_{2}+g_{1}\right)}{h_{1}+2 h_{2}+h_{3}+g_{1}+g_{2}} .
\end{aligned}
$$

(iv) The expressions for $m_{i}^{(4)}, n_{\imath}^{(4)}$ are the same as those in (iii) except that $b_{\jmath}$ is replaced by $c_{j}$ throughout.

(v) The expressions for $m_{i}^{(5)}, n_{i}^{(5)}$ are the same as those in (iii) except that $a_{j}$ is replaced by $c_{j}$ throughout.

We can also derive an implicit expression for the $L^{2}$ metric on the 8-dimensional manifold $M^{8}$.

We saw earlier that $M^{8}$ is topologically $\mathbf{R}^{5} \times S U(2) / \mathbf{Z}_{2}$, so global coordinates will not exist. We can take as local coordinates on $M^{8}$ the functions $\alpha_{1}, \alpha_{2}, \alpha_{3}, \alpha_{4}, \alpha_{5}$ of (41-45), together with the Euler angles $\alpha_{6}, \alpha_{7}, \alpha_{8}$, which satisfy

$$
\begin{aligned}
\cos \alpha_{6} & =\frac{\left\langle\hat{T}_{3}(3), \sigma_{3}\right\rangle}{2\left\|\hat{T}_{3}(3)\right\|}, \\
\cos \alpha_{7} & =\frac{-\left\langle\hat{T}_{3}(3), \sigma_{1}\right\rangle}{2\left\|\left[\hat{T}_{3}(3), \sigma_{3}\right]\right\|}, \\
\cos \alpha_{8} & =\frac{\left\langle\left[\hat{T}_{3}(3), \hat{T}_{1}(3)\right],\left[\sigma_{3}, \hat{T}_{3}(3)\right]\right\rangle}{\left\|\left[\hat{T}_{3}(3), \hat{T}_{1}(3)\right]\right\|\left\|\left[\sigma_{3}, \hat{T}_{3}(3)\right]\right\|}
\end{aligned}
$$

where $\left(\hat{T}_{0}, \hat{T}_{1}, \hat{T}_{3}, \hat{T}_{4}\right)$ are the Nahm matrices representing a point on $M^{8}$.

If we view the Nahm matrices at $t=3$ as vectors in $\mathbb{R}^{3}$, then $\alpha_{1}, \ldots, \alpha_{5}$ specify them up to an overall rotation. This rotation is parametrised by the Euler angles.

Note that $\alpha_{1}, \ldots, \alpha_{8}$ are invariant under the action of the gauge group $G_{0}^{0}$, so are well-defined on $M^{8}$.

As usual, we need to find tangent vectors $\hat{Y}\left(\alpha_{1}\right), \ldots, \hat{Y}\left(\alpha_{8}\right)$, at each point of $M^{8}$ dual to $d \alpha_{1}, \ldots, d \alpha_{8}$, and then compute their inner products to find the metric. The duality relations that the $\hat{Y}\left(\alpha_{j}\right)$ will satisfy are

$$
d \alpha_{\imath}\left(\hat{Y}\left(\alpha_{j}\right)\right)=\delta_{\imath j} ; \quad i, j=1, \ldots, 8
$$


These relations will be expressible as linear equations in $m_{i}, n_{i}$, and solving these equations will give us the tangent vectors $\hat{Y}\left(\alpha_{i}\right)$. Their inner products can then be calculated using Lemma 4.5.

Now a general point of $M^{8}$ will be of the form

$$
\begin{aligned}
\left(\hat{T}_{0},\right. & \left.\hat{T}_{1}, \hat{T}_{2}, \hat{T}_{3}\right) \\
= & \left(-E(t) \dot{A}(t) A(t)^{-1} E(t)^{-1}-\dot{E}(t) E(t)^{-1}, E(t) A(t) \sum a_{\imath} T_{i} A(t)^{-1} E(t)^{-1}\right. \\
& \left.E(t) A(t) \sum b_{i} T_{i} A(t)^{-1} E(t)^{-1}, E(t) A(t) \sum c_{i} T_{i} A(t)^{-1} E(t)^{-1}\right)
\end{aligned}
$$

where

$$
\begin{gathered}
T_{i}=\frac{1}{2} f_{i} \sigma_{1} \quad(i=1,2,3), \\
f_{1}^{2} \leq f_{2}^{2} \leq f_{3}^{2}, \\
E(t), A(t) \in C^{\omega}([0,3], S U(2)), \\
E(0)=I d, \quad E(3)=E \in S U(2),
\end{gathered}
$$

and

$$
A(0) \text { descends to } A=\left(\begin{array}{lll}
a_{1} & a_{2} & a_{3} \\
b_{1} & b_{2} & b_{3} \\
c_{1} & c_{2} & c_{3}
\end{array}\right) \in S O(3), \quad A(3)=I d \text {. }
$$

In other words, $\left(\hat{T}_{0}, \hat{T}_{1}, \hat{T}_{2}, \hat{T}_{3}\right)$ is obtained from $\left(0, \frac{1}{2} f_{1} \sigma_{1}, \frac{1}{2} f_{2} \sigma_{2}, \frac{1}{2} f_{3} \sigma_{3}\right)$ by applying the $S O(3)$ action of $A$ and the $S U(2)$ action of $E$ (recall from 3.3 that these two actions commute on $M^{8}$ ).

From 3.5 we see that a tangent vector $\hat{Y}$ at $\left(\hat{T}_{0}, \hat{T}_{1}, \hat{T}_{2}, \hat{T}_{3}\right)$ may be represented as

$$
\begin{aligned}
\hat{Y}= & \left(\hat{Y}_{0}, \hat{Y}_{1}, \hat{Y}_{2}, \hat{Y}_{3}\right) \\
= & \left(E(t) A(t) Y_{0} A(t)^{-1} E(t)^{-1}, E(t) A(t) \sum a_{i} Y_{i} A(t)^{-1} E(t)^{-1},\right. \\
& \left.E(t) A(t) \sum b_{i} Y_{i} A(t)^{-1} E(t)^{-1}, E(t) A(t) \sum c_{i} Y_{i} A(t)^{-1} E(t)^{-1}\right),
\end{aligned}
$$

where $Y=\left(Y_{0}, Y_{1}, Y_{2}, Y_{3}\right)$ is a tangent vector at $\left(T_{0}, T_{1}, T_{2}, T_{3}\right)=\left(0, \frac{1}{2} f_{1} \sigma_{1}, \frac{1}{2} f_{2} \sigma_{2}\right.$, $\left.\frac{1}{2} f_{3} \sigma_{3}\right)$ defined by parameters $m_{i}, n_{i}$ as in 4.1 .

Note that, using the obvious notation, $\int_{0}^{3}\langle\hat{Y}, \hat{Z}\rangle=\int_{0}^{3}\langle Y, Z\rangle$.

We have the following proposition.

Proposition 4.7. Let $\left(\hat{T}_{0}, \hat{T}_{1}, \hat{T}_{2}, \hat{T}_{3}\right) \in M^{8}$ be the image of a configuration $\left(0, \frac{1}{2} f_{1} \sigma_{1}\right.$, $\left.\frac{1}{2} f_{2} \sigma_{2}, \frac{1}{2} f_{3} \sigma_{3}\right)$ under the action of $A \in S O(3)$ and $E \in S U(2)$, given by the expression (62).

We can define tangent vectors $\hat{Y}\left(\alpha_{1}\right), \ldots, \hat{Y}\left(\alpha_{8}\right)$ at $\left(\hat{T}_{0}, \hat{T}_{1}, \hat{T}_{2}, \hat{T}_{3}\right)$ dual to $d \alpha_{1}, \ldots, d \alpha_{8}$, by the following prescription.

Let

$$
\begin{aligned}
\hat{Y}\left(\alpha_{j}\right)= & \left(E(t) A(t) Y_{0} A(t)^{-1} E(t)^{-1}, E(t) A(t) \sum a_{i} Y_{i} A(t)^{-1} E(t)^{-1},\right. \\
& \left.E(t) A(t) \sum b_{i} Y_{i} A(t)^{-1} E(t)^{-1}, E(t) A(t) \sum c_{i} Y_{i} A(t)^{-1} E(t)^{-1}\right),
\end{aligned}
$$


where $\left(Y_{0}, Y_{1}, Y_{2}, Y_{3}\right)$ is a tangent vector at $\left(0, \frac{1}{2} f_{1} \sigma_{1}, \frac{1}{2} f_{2} \sigma_{2}, \frac{1}{2} f_{3} \sigma_{3}\right)$ as in 4.1 defined by parameters $m_{i}, n_{i}$ satisfying

$$
\left(\begin{array}{c}
-2 m_{1} \\
-2 n_{1} \\
m_{2} \\
n_{3} \\
m_{4}-n_{4}
\end{array}\right)=R\left(A^{T}\right) e_{j}
$$

if $j \in\{1,2,3,4,5\}$

$$
\left(\begin{array}{c}
m_{1} \\
n_{1} \\
m_{2} \\
n_{3} \\
m_{4}-n_{4}
\end{array}\right)=0
$$

if $j \in\{6,7,8\}$, and

$$
\begin{array}{llll}
d \alpha_{6}\left(\hat{Y}\left(\alpha_{j}\right)\right)=1 & \text { if } j=6, & d \alpha_{6}\left(\hat{Y}\left(\alpha_{j}\right)\right)=0 & \text { otherwise, } \\
d \alpha_{7}\left(\hat{Y}\left(\alpha_{j}\right)\right)=1 & \text { if } j=7, & d \alpha_{7}\left(\hat{Y}\left(\alpha_{j}\right)\right)=0 & \text { otherwise, } \\
d \alpha_{8}\left(\hat{Y}\left(\alpha_{j}\right)\right)=1 & \text { if } j=8, & d \alpha_{8}\left(\hat{Y}\left(\alpha_{j}\right)\right)=0 & \text { otherwise. }
\end{array}
$$

Combining this with Lemma 4.5, which shows us how to calculate the inner products of tangent vectors, we obtain an expression for the metric.

Theorem 4.8. The $L^{2}$ metric on $M^{8}$ is given at $\left(\hat{T}_{0}, \hat{T}_{1}, \hat{T}_{2}, \hat{T}_{3}\right)$ by

$$
d s^{2}=\sum_{i, j=1}^{8} H_{\imath j} d \alpha_{i} d \alpha_{j}
$$

where

$$
H_{i j}=\sum_{k=1}^{4} m_{k}^{(i)} m_{k}^{(j)}\left(h_{1}+g_{1}\right)+n_{k}^{(i)} n_{k}^{(j)}\left(h_{3}+g_{2}\right)+\left(m_{k}^{(i)} n_{k}^{(j)}+m_{k}^{(j)} n_{k}^{(i)}\right) h_{2}
$$

and $\left\{m_{k}^{(j)}, n_{k}^{(\jmath)} ; k=1,2,3,4\right\}$ is the solution to the system of linear equations given in 4.7 .

\section{A Surface of Revolution}

The expression for the metric we have obtained is a complicated one involving elliptic functions and their integrals. However these functions will in some cases (Nahm data with axial symmetry) reduce to elementary trigonometric and hyperbolic functions. In this section we shall study the totally geodesic surface of revolution $\Sigma$ in $M^{8}$ introduced in 4.3. The points of $\Sigma$ represent $\mathrm{Nahm}$ data symmetric about a fixed axis in space and the metric on the surface is expressible in terms of trigonometric and hyperbolic functions. In [D1] we used this explicit expression for the metric to study geodesic flow on $\Sigma$, and, as mentioned in the introduction, used Manton's argument to make deductions about the dynamics of $S U(3)$ monopoles. 
Recall from 4.3 that we defined the curve $\Gamma$ in $M^{8}$ to be the family of Nahm data of the form

$$
\left(T_{0}, T_{1}, T_{2}, T_{3}\right)=\left(0, \frac{1}{2} f_{1} \sigma_{1}, \frac{1}{2} f_{2} \sigma_{2}, \frac{1}{2} f_{3} \sigma_{3}\right)
$$

where $f_{2}^{2}=f_{3}^{2}$.

This means that the functions $f_{i}$ are

$$
\left.\begin{array}{l}
f_{1}(t)=-D \operatorname{coth}(D t) \\
f_{2}(t)=-D \operatorname{cosech}(D t) \\
f_{3}(t)=-D \operatorname{cosech}(D t)
\end{array}\right\}
$$

for $D>0$, or

$$
f_{1}(t)=f_{2}(t)=f_{3}(t)=-1 / t
$$

or

$$
\left.\begin{array}{l}
f_{1}(t)=-D \cot (D t) \\
f_{2}(t)=-D \operatorname{cosec}(D t) \\
f_{3}(t)=-D \operatorname{cosec}(D t)
\end{array}\right\}
$$

for $0<D<\pi / 3$.

Note that the condition $D<\pi / 3$ in the last example is necessary because one of the conditions we have imposed on our Nahm data is that it should be analytic on the interval $(0,3]$.

Consider the $U(1)$ subgroup

$$
\left\{\left(\begin{array}{cc}
e^{i \theta} & 0 \\
0 & e^{-i \theta}
\end{array}\right): \theta \in[0,2 \pi]\right\}
$$

of $S U(2)$.

We defined $\Sigma$ to be the union of the orbits of this $U(1)$ containing points of $\Gamma$. Therefore $\Sigma$ is a surface of revolution in $M^{8}$. Moreover, we proved in 4.3 that $\Sigma$ was totally geodesic by identifying it as the fixed point set of a subgroup of the isometry group of $M^{8}$.

We could take $\alpha_{1}=\left\langle T_{1}, T_{1}\right\rangle-\left\langle T_{2}, T_{2}\right\rangle$ as a coordinate on $\Gamma$, but it is convenient to introduce coordinates $\nu, \kappa$ as follows. If $\alpha_{1} \geq 0$, let $\nu=-3 \sqrt{\alpha_{1}}$; if $\alpha_{1} \leq 0$, let $\kappa=3 \sqrt{-\alpha_{1}}$. Therefore

$$
\begin{aligned}
& \begin{array}{l}
\text { represents the solution given by (66) with } D=-\frac{\nu}{3}, \\
\nu=0, \kappa=0 \\
\text { represents solution (67), } \\
\text { represents the solution given by (68) with } D=\frac{\kappa}{3} .
\end{array} \\
& \text { ren }
\end{aligned}
$$

We shall refer to the part of $\Sigma$ representing hyperbolic and spherically symmetric $\mathrm{Nahm}$ data as region 1, and to the part representing trigonometric and spherically symmetric solutions as region 2 . Letting $\theta$ denote the $U(1)$ coordinate, we can take $\nu, \theta$ as coordinates on region 1 , and $\kappa, \theta$ as coordinates on region 2 .

Let us first work in region 1 . The $U(1)$ symmetry of $\Sigma$ means that we need only consider points of $\Sigma$ representing Nahm data in diagonal form.

A basis for the tangent space to $\Sigma$ at each point in this region is provided by the vector field $Y(\nu)$ dual to the coordinate $\nu$, and the Killing vector field $Y(\theta)$ generated by the $U(1)$ action. We shall calculate the metric with respect to these vector fields. 
Now the tangent vector $Y(\nu)$ dual to $\nu$ is given by

$$
Y(\nu)=\frac{2}{9} \nu\left(Y\left(\alpha_{1}\right)+Y\left(\alpha_{2}\right)\right)
$$

where $Y\left(\alpha_{1}\right)$ and $Y\left(\alpha_{2}\right)$ are the vector fields calculated in Sect. 4. Using Lemma 4.5, and substituting in our expressions for the $f_{i}$, we find that the coefficient of the $d \nu^{2}$ term of the metric is

$$
\int_{0}^{3}\|Y(\nu)\|^{2}=-\frac{1}{3} \nu \operatorname{cosech}^{2} \nu \operatorname{coth} \nu\left(\frac{\sinh 2 \nu}{2 \nu}-1\right)^{2}+\frac{\sinh 2 \nu}{6 \nu}-\frac{1}{3} .
$$

Similarly, in region 2 of $\Sigma$, parametrized by $\kappa, \theta$ we find

$$
\int_{0}^{3}\|Y(\kappa)\|^{2}=-\frac{1}{3} \kappa \operatorname{cosec}^{2} \kappa \cot \kappa\left(\frac{\sin 2 \kappa}{2 \kappa}-1\right)^{2}+\frac{1}{3}-\frac{\sin 2 \kappa}{6 \kappa}
$$

where $Y(\kappa)$ is dual to $\kappa$.

Observe that the elliptic functions of the expression for the metric on $M^{8}$ have reduced to hyperbolic and trigonometric functions in region 1 and region 2 respectively.

We now need to calculate the $d \theta^{2}$ term of the metric on $\Sigma$ (recall $\theta$ is the $U(1)$ coordinate) which is given by the length squared of the vector field generated by the $U(1)$ action. Let us first find this vector field explicitly.

The vector field obtained by differentiation of the action of $U(1)$ takes the value $X=\left(X_{0}, X_{1}, X_{2}, X_{3}\right)$ at $\left(0, \frac{1}{2} f_{1} \sigma_{1}, \frac{1}{2} f_{2} \sigma_{2}, \frac{1}{2} f_{3} \sigma_{3}\right)$, where

$$
X_{0}=\frac{2}{3}\left(\begin{array}{l}
1 \\
0 \\
0
\end{array}\right), \quad X_{1}=\left(\begin{array}{l}
0 \\
0 \\
0
\end{array}\right), \quad X_{2}=\frac{2}{3}\left(\begin{array}{c}
0 \\
0 \\
-t f_{2}
\end{array}\right), \quad X_{3}=\frac{2}{3}\left(\begin{array}{c}
0 \\
t f_{3} \\
0
\end{array}\right) \text {. }
$$

Now $X$ is not orthogonal to the orbit of the gauge group $G_{0}^{0}$ through $\left(T_{0}, T_{1}, T_{2}, T_{3}\right)$, so is not a tangent vector to $\Sigma$ (or even $M^{8}$ ). Our Killing field $Y(\theta)$ on $\Sigma$ is the orthogonal projection of $X$ onto the tangent space to $\Sigma$.

Referring to the formula above for $X$, and to our explicit expressions for the tangent vectors to $\Sigma$, we arrive at the following expression for the $d \theta^{2}$ term of the metric.

In region 1

$$
\int_{0}^{3}\|Y(\theta)\|^{2}=\frac{4 \operatorname{cosech}^{4} \nu\left(\frac{3 \sinh 2 \nu}{2 \nu}-3\right)^{2}}{-\frac{3}{\nu} \operatorname{cosech}^{2} \nu \operatorname{coth} \nu\left(\frac{3 \sinh 2 \nu}{2 \nu}-3\right)^{2}+\frac{9}{\nu^{2}}\left(\frac{3 \sinh 2 \nu}{2 \nu}-3\right)}
$$

and in region 2

$$
\int_{0}^{3}\|Y(\theta)\|^{2}=\frac{4 \operatorname{cosec}^{4} \kappa\left(3-\frac{3 \sin 2 \kappa}{2 \kappa}\right)^{2}}{-\frac{3}{\kappa} \operatorname{cosec}^{2} \kappa \cot \kappa\left(\frac{3 \sin 2 \kappa}{2 \kappa}-3\right)^{2}+\frac{9}{\kappa^{2}}\left(3-\frac{3 \sin 2 \kappa}{2 \kappa}\right)} .
$$


We also find that the $d \nu d \theta$ and $d \kappa d \theta$ terms vanish.

Now $-I d \in S U(2)$ acts trivially on $\Sigma$, so we shall take $\phi=2 \theta$ as our angular parameter.

After making this reparametrisation, and simplifying our expressions further, we finally obtain the following form of the metric on $\Sigma$.

Theorem 5.1. The metric on the totally geodesic surface $\Sigma$ is

$$
\begin{aligned}
d s^{2}= & (\nu-\sinh \nu \cosh \nu) \\
& \times\left(-\frac{\cosh \nu}{3 \nu \sinh ^{3} \nu}(\nu-\tanh \nu) d \nu^{2}+\frac{\nu d \phi^{2}}{3 \cosh \nu \sinh \nu(\tanh \nu-\nu)}\right)
\end{aligned}
$$

in region 1 , and

$$
\begin{aligned}
d s^{2}= & (\kappa-\sin \kappa \cos \kappa) \\
& \times\left(-\frac{\cos \kappa}{3 \kappa \sin ^{3} \kappa}(\kappa-\tan \kappa) d \kappa^{2}+\frac{\kappa d \phi^{2}}{3 \cos \kappa \sin \kappa(\tan \kappa-\kappa)}\right)
\end{aligned}
$$

in region 2 .

Remember that $\nu \leq 0$ and $0 \leq \kappa<\pi$.

We have calculated the metric on the totally geodesic surface $\Sigma$, and we can use this to get a detailed picture of the geometry of $\Sigma$. First we shall check that the expressions we have for the metric on region 1 and region 2 fit together in a smooth fashion at $\nu=\kappa=0$. Recall that region 1 of $\Sigma$ represents hyperbolic Nahm data, while region 2 represents trigonometric Nahm data, in the terminology of Sect. 3. The circle defined by $\nu=0$ on $\Sigma$ corresponds to spherically symmetric Nahm data.

Let us write the metric as $d s^{2}=A_{1}(\nu) d \nu^{2}+B_{1}(\nu) d \phi^{2}$ in region 1 and $d s^{2}=A_{2}(\kappa) d \kappa^{2}+B_{2}(\kappa) d \phi^{2}$ in region 2 .

It can easily be seen that

$$
A_{1}(\nu) d \nu^{2}+B_{1}(\nu) d \phi^{2}=A_{2}(i \nu) d(i \nu)^{2}+B_{2}(i \nu) d \phi^{2} .
$$

Recalling that $\nu=-3 \sqrt{\alpha_{1}}$ for $\alpha_{1} \geq 0$ and $\kappa=3 \sqrt{-\alpha_{1}}$ for $\alpha_{1} \leq 0$, and expressing $A_{1}, A_{2}, B_{1}, B_{2}$ as functions of the coordinate $\alpha_{1}$, we find that the expressions for the metric fit together smoothly at $\nu=0$.

Our formula for the metric has a coordinate singularity at $\nu=0$, where $A_{1}(\nu)$ vanishes. This singularity is removed by the reparametrisation $\alpha_{1}=\frac{\nu^{2}}{9}$.

The functions $A_{1}, A_{2}, B_{1}, B_{2}$ have the following properties.

(i) $A_{1}(\nu)$ is monotonic decreasing for $\nu<0$ and $A_{2}(\kappa)$ is monotonic increasing for $0<\kappa<\pi, A_{1}(0)=A_{2}(0)=0$ and $A_{2}(\kappa)$ tends to infinity as $\kappa$ tends to $\pi$.

(ii) $B_{1}(\nu), B_{2}(\kappa)$ are monotonic increasing on $\nu<0,0<\kappa<\pi$ respectively. $B_{2}(\kappa)$ tends to infinity as $\kappa$ tends to $\pi$.

Recall that the boundary of $\Sigma$ given by $\kappa=\pi$ represents Nahm data with a pole at $t=3$, which corresponds via the Nahm transform to $S U(2)$-monopoles. This boundary is not included in the moduli space $M^{8}$. A natural question to ask is whether our metric extends over the boundary; if this were the case, the metric on $M^{8}$ would be incomplete. We see from the expressions for the metric that the $d \kappa^{2}$ and $d \phi^{2}$ terms have triple and simple poles respectively at $\kappa=\pi$ and hence the metric fails to extend over the boundary. 
Let us consider the asymptotic behaviour of the metric as $\nu$ tends to $-\infty$ and as $\kappa$ tends to $\pi$. Letting $\nu$ tend to $-\infty$ corresponds to considering hyperbolic solutions $f_{1}(t)=-D \operatorname{cosech}(D t)$ etc. with $D$ tending to $\infty$.

It is easily seen from our expressions for the metric that $A_{1}(\nu), B_{1}(\nu) \rightarrow 1 / 3$ as $\nu \rightarrow-\infty$. Therefore the metric is asymptotically $\frac{1}{3}\left(d \nu^{2}+d \phi^{2}\right)$, that is, a cylinder of radius $\frac{1}{\sqrt{3}}$.

We can perform a similar analysis in the case $\kappa \rightarrow \pi$. Letting

$$
r=2 \sqrt{\frac{\kappa}{3(\pi-\kappa)}}
$$

the metric becomes asymptotically

$$
d r^{2}+\frac{1}{4} r^{2} d \phi^{2}
$$

as $r \rightarrow \infty$. This means that the surface $\Sigma$ is asymptotically a cone of vertex angle $\pi / 3$.

Our surface $\Sigma$, with the metric we have calculated, is a surface of revolution. Using the standard expressions for the radius and curvature of such a surface, and examining the behaviour of the coefficients $A_{1}, A_{2} B_{1}, B_{2}$ of the metric, leads us to the following conclusions. We take $\gamma \in(-\infty, \infty)$ to be a coordinate on $\Sigma$ orthogonal to the $U(1)$ direction, such that $\gamma \leq 0$ in region 1 and $\gamma \geq 0$ in region 2 .

Theorem 5.2. The totally geodesic surface $\Sigma$ has negative curvature and monotonic increasing radius. The radius tends to $\frac{1}{\sqrt{3}}$ as $\gamma$ tends to $-\infty$ and tends to $\infty$ as $\gamma$ tends to $\infty$. As $\gamma \rightarrow-\infty, \Sigma$ is asymptotically a cylinder of radius $\frac{1}{\sqrt{3}}$, and as $\gamma \rightarrow \infty, \Sigma$
is asymptotically a cone of vertex angle $\pi / 3 . \quad \square$

\section{Hyperkähler Quotients}

We have encountered the hyperkähler 8-manifold $M^{8}$ as a hyperkähler quotient of $M^{12}$ by a triholomorphic circle action. Now, as explained in Sect. 3, there is a triholomorphic action of $S U(2)$ on $M^{8}$, so there will be an induced moment map from $M^{8}$ to $\mathbb{R}^{3} \otimes \mathfrak{s u}(2)$. In particular, we may restrict ourselves to considering the action of a $U(1)$ subgroup of $S U(2)$ on $M^{8}$; this will give rise to a moment map $\mu$ taking values in $\mathbb{R}^{3}$. In this way we obtain a hyperkähler 4-manifold $\mu^{-1}\left(\lambda_{1}, \lambda_{2}, \lambda_{3}\right) / U(1)$ for each triple $\left(\lambda_{1}, \lambda_{2}, \lambda_{3}\right)$ in $\mathbb{R}^{3}$. As a first step towards understanding these 4-manifolds, let us explicitly calculate the moment map $\mu$ associated to a circle subgroup of $S U(2)$.

Theorem 6.1. Consider the U(1) subgroup of $S U(2)$ which fixes $\sigma_{2}$ when $S U(2)$ acts on $\mathfrak{s u ( 2 )}$ in the adjoint representation (this subgroup is just $S O(2, \mathbb{C})$ ).

The moment map $\mu: M^{8} \mapsto \mathbb{R}^{3}$ given by this $U(1)$ action is

$$
\mu:\left(T_{0}, T_{1}, T_{2}, T_{3}\right) \mapsto\left(\left\langle T_{1}(3), \sigma_{2}\right\rangle,\left\langle T_{2}(3), \sigma_{2}\right\rangle,\left\langle T_{3}(3), \sigma_{2}\right\rangle\right) .
$$

Proof. This is a straightforward (though long) calculation along the lines of 3.7. Differentiation of the action of the $U(1)$ subgroup at $\theta=0$ gives the vector field $X$ generated by the action. Using the formulae (15) we can calculate $\omega_{i}(X, Y)(i=$ 
1,2,3), where $\omega_{i}(i=1,2,3)$ are the Kähler forms on $M^{8}$ and $Y$ is a tangent vector to $M^{8}$. We find that

$$
\omega_{i}(X, Y)=\left\langle Y_{i}(3), \sigma_{2}\right\rangle \quad(i=1,2,3) .
$$

The result follows.

Note that $\mu$ is invariant under the action of the gauge group $G_{0}^{0}$ and of the centre of $U(2)$, so is well-defined on the moduli space $M^{8}$.

Corollary 6.2. For each $\left(\lambda_{1}, \lambda_{2}, \lambda_{3}\right) \in \mathbb{R}^{3}, \mu^{-1}\left(\lambda_{1}, \lambda_{2}, \lambda_{3}\right) / U(1)$ is a hyperkähler 4-manifold.

Proof. This follows from Theorem 2.4 and the freeness of the $U(1)$ action.

Definition 6.3. Let us denote $\mu^{-1}\left(2 \lambda_{1}, 2 \lambda_{2}, 2 \lambda_{3}\right) / U(1)$ by $M\left(\lambda_{1}, \lambda_{2}, \lambda_{3}\right)$.

We have produced a family of hyperkähler 4-manifolds. By virtue of the hyperkähler property, they are self-dual and Ricci-flat, and so are of interest in physics as examples of gravitational instantons.

It follows from our expression for the moment map $\mu$, and from the description of our 4-manifolds as quotients of level sets of $\mu$, that the $S O(3)$ action on $M^{8}$ gives an isometry between $M\left(\lambda_{1}, \lambda_{2}, \lambda_{3}\right)$ and $M(0,0, \lambda)$, where $\lambda_{1}^{2}+\lambda_{2}^{2}+\lambda_{3}^{2}=\lambda^{2}$. Consequently, we need only consider $M(0,0, \lambda)$, where $\lambda \geq 0$.

We see that the full group $S O(3)$ acts isometrically on $M(0,0,0)$, whereas only the $S^{1}$ subgroup of $S O(3)$ given by

$$
\left\{\left(\begin{array}{ccc}
\cos \theta & \sin \theta & 0 \\
-\sin \theta & \cos \theta & 0 \\
0 & 0 & 1
\end{array}\right): \theta \in[0,2 \pi]\right\}
$$

acts on $M(0,0, \lambda)$ for $\lambda \neq 0$.

In fact we can identify $M(0,0,0)$ with a known gravitational instanton, as follows.

Proposition 6.4. $M(0,0,0)$ is isometric, up to a constant scale factor, to the double cover of the moduli space of centred, charge two, $S U(2)$ monopoles.

Proof. It easily follows from our description of $\mu$ and the properties of Jacobi elliptic functions that $\left(T_{0}, T_{1}, T_{2}, T_{3}\right)$ lies in $\mu^{-1}(0,0,0)$ if and only if it extends to a solution to the Nahm equations with the boundary conditions of 2.2 on the interval $[0,6]$. Restriction of Nahm matrices from $[0,6]$ to $[0,3]$ gives us a diffeomorphism from the moduli space of Nahm data for centred, charge two, $S U(2)$ monopoles onto a quotient by $\mathbf{Z}_{2}$ of $M(0,0,0)$. Moreover this map is (up to a constant scale factor) an isometry. Hurtubise [Hu2] and Nakajima [Na] have shown that the Nahm transform for $S U(2)$ monopoles is an isometry, so the proposition is proved.

Corollary 6.5. $M(0,0,0)$ is complete and homeomorphic to the double cover of $S^{4}-\mathbb{R} \mathbb{P}^{2}$. It has the homotopy type of $S^{2}$.

Proof. This follows from the results of $[\mathrm{AH}]$ and [Hu1] on the completeness and topology of the $S U(2)$ monopole moduli space.

We see, therefore, that we have a one-parameter family of gravitational instantons arising as deformations of the $S U(2)$ monopole moduli space studied by Atiyah and Hitchin. In a future paper [D2] we shall show that the moduli spaces $M^{12}$ and $M^{8}$ are isomorphic as complex manifolds to spaces of based rational maps from $\mathbb{C P} \mathbb{P}^{1}$ to $\mathbb{C P}^{2}$. 
We shall use this description to express the hyperkähler four-manifolds $M(0,0, \lambda)$ as hypersurfaces in $\mathbb{C}^{3}$, and make more precise the sense in which they are deformations of the Atiyah-Hitchin manifold.

Acknowledgements. I am grateful to Professor Nigel Hitchin for useful discussions, to the S.E.R.C. for a research studentship, and to Peterhouse, Cambridge for a Stone Research Fellowship.

\section{References}

[AH] Atiyah, M.F., Hitchin, N.J.: The geometry and dynamics of magnetic monopoles. Princeton, NJ: Princeton University Press 1988

[C] Calabi, E.: Métriques Kahlériennes et fibrés holomorphes. Ann. Sci. École. Norm. Sup. 12, 269-294 (1979)

[CG] Corrigan, E., Goddard, P.: Construction of instanton and monopole solutions and reciprocity. Ann. Phys. 154, 253-279 (1984)

[D1] Dancer, A.S.: Nahm data and SU(3) monopoles. Nonlinearity 5, 1355-1373 (1992)

[D2] Dancer, A.S.: A family of hyperkähler manifolds. (To appear in Quarterly Journal of Mathematics)

[H1] Hitchin, N.J.: On the construction of monopoles. Commun. Math. Phys. 89, 145-190 (1983)

[H2] Hitchin, N.J.: Monopoles, minimal surfaces and algebraic curves. Montréal: Les Presses de L’Université de Montréal 1987

[HKLR] Hitchin, N.J., Karlhede, A., Lindström, U., Rǒcek, M.: Hyperkähler metrics and supersymmetry. Commun. Math. Phys. 108, 535-589 (1987)

[Hul] Hurtubise, J.: $S U(2)$ monopoles of charge 2. Commun. Math. Phys. 92, 195-202 (1983)

[Hu2] Hurtubise, J.: Metric properties of monopole moduli spaces. Abstracts. Am. Math. Soc. 11, No. 3, 268 (1990)

[M] Manton, N.S.: A remark on the scattering of BPS monopoles. Phys. Lett. B 110, 54-56 (1982)

[N] Nahm, W.: The construction of all selfdual monopoles by the ADHM method. In: Craigie, N.S., Goddard, P., Nahm, W. (eds.), Monopoles in quantum field theory. Singapore: World Scientific 1982

[Na] Nakajima, H.: Monopoles and Nahm's equations. University of Tokyo preprint, 1991

[WW] Whittaker, E., Watson, G.N.: A course of modern analysis. Cambridge: Cambridge University Press 1927

Communicated by S.-T. Yau 